\title{
La intervención social del Estado durante el gobierno de Cambiemos en Argentina. El caso del Programa Hacemos Futuro*
}

\author{
Agustín Zuccaro*** \\ Fernando-Gabriel Laya**** \\ Universidad Nacional de La Plata, Argentina \\ https://doi.org/10.15446/frdcp.n21.87812
}

\section{Resumen}

El objetivo del artículo es caracterizar la intervención social del Estado durante el gobierno de Cambiemos a partir de la puesta en funcionamiento del Programa "Hacemos Futuro". En este sentido, el trabajo expone los resultados de un informe técnico donde se analizaron tanto los aspectos formales y normativos, como el proceso de implementación del mencionado programa entre 2018-2019. Se trata de un abordaje cualitativo que fue viabilizado a partir del análisis del discurso, en función del cual surge como

\footnotetext{
*Artículo recibido: $1^{\circ}$ de febrero de 2021 / Aceptado: 3 de agosto de 2021 / Modificado: 15 de agosto de 2021. El artículo es producto, por un lado, de un informe técnico realizado durante el año 2019 en el marco de la Dirección de Gestión de Políticas Públicas de la Prosecretaría de Políticas Sociales de la Universidad Nacional de La Plata (Argentina) y, por el otro, de la investigación denominada "Partidos políticos, hegemonía y poder. El caso de la construcción política del PRO (2002-2019)", la cual se encuentra en curso y se lleva adelante en el marco de una beca doctoral otorgada por el Consejo Nacional de Investigaciones Científicas y Técnicas (Conicet), Argentina.

** Licenciado en Trabajo Social por la Universidad Nacional de La Plata, Argentina. Becario doctoral del Conicet en la Universidad Nacional de La Plata, Facultad de Trabajo Social, Instituto de Estudios en Trabajo Social y Sociedad. Correo electrónico: aguszuccaro@gmail.com iD https://orcid.org/0000-0002-2919-6305 **** Licenciado en Sociología por la Universidad Nacional de La Plata, Argentina. Estudiante de doctorado en Ciencias Sociales de la Universidad Nacional de La Plata. Correo electrónico: fglaya@gmail.com (iD) https://orcid.org/0000-0003-0167-4165
}

Zuccaro, A. y Laya, F. G. (2022). La intervención social del Estado durante el gobierno de Cambiemos en Argentina. El caso del Programa Hacemos Futuro. FORUM. Revista Departamento Ciencia Política, 21, 143-165. https://doi.org/10.15446/frdcp.n21.87812 
principal conclusión la formulación de la pobreza como el "locus" de la cuestión social y una adopción del abordaje estatal que buscó construir un andamiaje articulador entre la perspectiva del mercado y la gestión gubernamental de la asistencia.

Palabras clave: políticas públicas; Cambiemos; Hacemos Futuro; cuestión social; Estado.

\title{
The Social Intervention of the State during the Cambiemos Government in Argentina. The Case of the We Make Future Program
}

\begin{abstract}
The objective of the article is to characterize the social intervention of the State during the Cambiemos government from the start of the "Make the Future" Program. In this sense, the work presents the results of a technical report where both the formal and regulatory aspects, as well as the implementation process of the aforementioned program, between 2018-2019, were analyzed. It is a qualitative approach that was made viable based on discourse analysis, in function of which a formulation of poverty as the "locus" of the social issues emerges as the main conclusion and an adoption of the state's approach that sought to build an articulating scaffold between the market perspective and government management of aid.
\end{abstract}

Keywords: public policies; Cambiemos; Hacemos Futuro; social issue; State.

\section{Introducción}

La relación siempre dinámica y, también frecuentemente problemática, entre las elites y el Estado conforma un objeto de estudio nodal en las investigaciones recientes en América Latina. El "giro a la derecha" en la región luego de la oleada posneoliberal, llamó la atención de diversos intelectuales, académicos y ensayistas que tuvieron como objetivo explicar sus motivos, pero también sus posibles consecuencias. En este marco, florecieron nuevamente -otro periodo fue la década de 1990- numerosos trabajos que abordaron desde distintos puntos de partida, así como con diversos clivajes de profundidad, la relación entre las elites y el Estado.

Durante el siglo XX el concepto de elites fue adquiriendo cada vez mayor relevancia, aunque se puede notar cierta intermitencia en las investigaciones latinoamericanas ${ }^{1}$ (Atria et al., 2017; Castellani, 2018; Gené, 2014). La gran mayoría de escritos sobre el

1. En Argentina, uno de los pioneros en estos estudios fue De Imaz (1964), quien postuló a las elites a partir de las posiciones ocupadas en las instituciones generales de la sociedad. 
tema, desde distintas ópticas, tienen un hilo conductor: destacan el hecho de que hay una minoría que manda y una mayoría que obedece (Mosca, 1984; Pareto, 1968). Dicho de otra forma, una elite es un pequeño grupo que concentra ciertos medios o bienes, es decir, un capital determinado - en el sentido que lo trabaja Bourdieu (1990; 2011) -, en relación a otro que no lo tiene. Por ello, se habla de elites económicas, profesionales, políticas, etcétera, aunque en muchos casos recorren circuitos similares de socialización, en donde tienen la posibilidad de ejercer influencia entre ellas (Mills, 1957).

En el caso argentino la alianza Cambiemos conformada por Propuesta Republicana (PRO), la Unión Cívica Radical (UCR) y la Coalición Cívica (CC-ARI), en 2015 llegó a ocupar posiciones de gobierno nacional por la tradición de elecciones libres. Un evento inédito en el país: un partido de derecha o centro-derecha asumía la conducción del Estado mediante el voto popular. La asunción de Mauricio Macri y la de su gabinete el 10 de diciembre de ese año, vino a combinar personificaciones específicas de trayectorias en elites económicas a la escena de las elites de la política nacional. Así, rápidamente distintos autores comenzaron a caracterizarlo como el gobierno de los managers, de los CEO's o Ceocracia, dada la gran cantidad de cuadros de mediana y alta gerencia que se incluyeron al gabinete presidencial (Canelo, 2019; Canelo y Castellani, 2017; Gené, 2018; Gessaghi, Landau y Luci, 2020; Vommaro, 2017).

En otro orden de trabajos e investigaciones recientes respecto al fenómeno de Cambiemos, varios son los abordajes que lo caracterizan como reversiones del neoliberalismo vinculado a la década de los años de 1990 en el país. En esa reedificación neoliberal, se señalaron similitudes y rupturas respecto al mencionado periodo a partir de análisis que lo situaban desde la clase, las afinidades ideológicas e inscripciones identitarias (Ansaldi, 2017; Natanson, 2018; Nazareno, 2019; Soler, Giordano y Saferstein, 2018; Vommaro, 2017; Vommaro, Morresi y Bellotti, 2015; Zícari, 2016).

En este marco más general, también cabe mencionar las producciones académicas que indagaron diversas áreas de gestión en la ciudad Autónoma de Buenos Aires (CABA) conducidas por el PRO entre 2007 y 2015 (García y Santiago, 2017; Landau, 2015; Salerno, 2017, 2019). En este mismo sentido, otra batería de abordajes, centran su mirada en el periodo 2015-2019 indagando cuestiones vinculadas a los principales cambios en materia de asistencia estatal que empleó la coalición gobernante, donde se remite un giro de un eje productivo al de la capacitación (Arcidiácono y Bermúdez, 2018; Ferrari-Mango y Campana, 2018; Hopp, 2018; Hudson, 2018; Ferrari-Mango, 2019; Voria y Míguez, 2019).

En este escenario, es que se busca incluir el presente artículo, con la motivación puesta en analizar el Programa Hacemos Futuro (PHF), a partir de un conjunto de repertorios enunciativos e interpretativos sobre el proceso de asistencia social radicado en la proyección de la intervención social del Estado. 
En términos generales, el PHF es un programa de transferencia condicionada de ingresos destinado a personas que tengan entre 18 y 65 años de edad, que como todos los que se catalogan bajo esa nomenclatura encuentra su razón de ser en la percepción de un subsidio mediante una contraprestación. En particular, pretende la finalización de los estudios primarios y/o secundarios, la realización de cursos de capacitación en oficios y la actualización cuatrimestral de datos. Así, una de las distinciones que establece el programa es la siguiente: la diferenciación entre quienes terminaron sus estudios y quienes no. Aquellas personas que se encuentren realizando estudios primarios o secundarios debían acreditar haber cumplido, o estar cumpliendo, con 64 horas anuales más en alguno de los cursos de formación validados por el Ministerio de Desarrollo Social ${ }^{2}$. Por su parte, quienes hayan terminado sus estudios secundarios deberían justificar 120 horas anuales. A partir del año 2019 las cargas horarias aumentaron a 120 y 300 horas, respectivamente.

De acuerdo a los objetivos expositivos, el artículo se articula de la siguiente manera. En primer lugar, se dedica una especial atención a la formulación de los criterios metodológicos y las fuentes que se utilizan para construir los datos. Luego, se aborda las cuestiones vinculadas a las definiciones generales que se usan para recortar la mirada y la perspectiva general de análisis. En tercera instancia, se analizan los discursos de la Ministra de Desarrollo Social buscando interpretar las definiciones del problema social estatizado y las formas que se apuntaron para solucionarlo durante el gobierno de Cambiemos. Es decir, caracterizar la razonabilidad gubernamental desde la cual se analizan los fenómenos y se delimitan estrategias de intervención. En un cuarto momento, se presta especial atención a la hermenéutica realizada por referentes de organizaciones sociales, investigadores y titulares del PHF, en relación a la puesta en marcha de esta política pública. Finalmente, se realiza una serie de consideraciones finales sobre lo detallado a lo largo de todo el texto.

\section{Sobre lo metodológico}

La elaboración de este artículo parte de la experiencia como integrantes del equipo de trabajo de la Dirección de Gestión de Políticas Públicas de la Prosecretaría de Políticas Sociales de la Universidad Nacional de La Plata (Argentina). En este marco es que se realiza un informe del PHF a partir del análisis tanto de sus aspectos formales y

\footnotetext{
2. Para simplificar se dirá Ministerio de Desarrollo Social. Sin embargo, conviene aclarar que luego se le iba a sumar a esta cartera la nomenclatura institucional "y Salud", cuando a partir de las recomendaciones del Fondo Monetario Internacional se redujo abruptamente el presupuesto de algunas áreas y el Ministerio de Salud se transformó en una secretaria, que dependía del lugar que comandaba Carolina Stanley.
} 
normativos, como del proceso de implementación recuperando distintas voces y saberes: titulares del programa, referentes de organizaciones sociales e investigadores especializados en la temática.

Para ello, se utiliza la propuesta metodológica Investigación + Gestión $(\mathrm{I}+\mathrm{G})$ :

I $+\mathrm{G}$ es el proceso mediante el cual un equipo de trabajo que tiene a cargo la gestión de política pública, se compromete a sostener un espacio de reflexión sistematizada, con un diseño metodológico que permita garantizar en simultáneo, la producción de conocimiento científico para retroalimentar y ajustar sus propios diseños de política sustantiva en el periodo temporal en el cual el equipo es responsable (Bonicatto, 2019, p. 50).

Como parte externa al PHF, es decir, sin tener vinculación formal con el Ministerio de Desarrollo Social de la Nación, pero sí una referencia institucional con los titulares del programa se combinan tres estrategias de recolección de datos. La primera, consistió en jornadas de difusión e información sobre el nuevo programa. En ella, se llevó adelante ocho encuentros donde participaron ciento treinta personas, en las cuales se articuló con trabajadores de la dependencia institucional del Estado para fortalecer el diálogo, despejar dudas y caracterizar los nuevos requisitos para su acceso, permanencia o suspensión.

Luego, en esta misma línea, se realizaron dos instancias de encuentro, donde participaron treinta y tres personas que tenían vinculación como "destinatarios" de la política pública. En este sentido, se configuraron los grupos focales en cantidades reducidas para indagar las interpretaciones y evaluaciones que realizaban del programa desde su propia experiencia. Esto es, las inquietudes, facilidades y barreras que se le presentaban para el acceso al ejercicio de los derechos.

Finalmente, se llevó adelante un encuentro con quince especialistas en la temática que se forjó en una mesa redonda de debate, diálogo y análisis sobre el PHF. La misma fue una propuesta abierta a la comunidad y se constituyó como una jornada académica, lo que permitió entrelazar claves analíticas e interpretativas de la investigación que se encontraba en curso. De la misma manera, esta vez en un encuentro cerrado, se realizó un espacio con referentes de organizaciones sociales de la ciudad de La Plata, con quienes se trabajó con la misma dinámica que la anterior. Una mesa redonda que tuvo un momento de exposición y otro de discusión e intercambio de experiencias.

Asimismo, se analizó y observó las declaraciones de Carolina Stanley, Ministra de Desarrollo Social de la Nación Argentina en el periodo 2015-2019. El motivo de la inclusión de estos relatos estuvo vinculado a poner atención a aquellos elementos y fundamentos simbólicos que funcionaron en el programa, más allá de los explicitados 
en su resolución formal. Este abordaje, resultó conveniente dado que fue quien presentó públicamente las cuestiones relativas a la intervención social del Estado durante el gobierno de Cambiemos. Dicho de otra forma, la intención fue reconstruir esa razonabilidad gubernamental que guió el diseño del programa a partir de los propios soportes explicativos que se utilizaron a fin de dar cuenta del problema que construyó y los objetivos que persiguió.

Este momento, se argumentó en la recolección de datos de fuentes secundarias, esto es, entrevistas en medios de comunicación. En relación con ello, podrían realizarse dos aclaraciones preliminares: en primer lugar, que los discursos analizados se ubican dentro de lo que se podría denominar como lenguaje político sobre la gestión estatal, es decir, que emerge como un dialecto específico en el marco de las construcciones simbólicas, sobre las cuales se monta una racionalidad y un ejercicio hermenéutico singular; luego, en segundo término, que son entrevistas en medios de comunicación, esto significa, que son discursos públicos, dado que se inscriben en el campo semántico de la formaciones de opinión pública y que tienen cierta circulación en el espacio social. Se considera que este análisis se constituyó como un relevamiento sustancial, para la comprensión de los principales argumentos que se tuvieron para llevar adelante el PHF y la interpretación de los fundamentos simbólicos sobre los cuáles se materializó.

En tal sentido, se sitúa esta perspectiva en el marco de los análisis de discurso, que considera que el lenguaje se constituye como un elemento analizador y como intermediador entre las estructuras sociales y las capacidades de agencia de los sujetos (Foucault, 1968, 2004; Vasilachis de Gialdino, 1997, 2005). De tal forma, se considera que el discurso es una práctica constituyente del mundo social, en donde subyacen los sentidos sociales que se configuran a partir de las hermenéuticas particulares. Las narrativas se materializan en un devenir que habla de intencionalidades, de construcciones $y$, fundamentalmente, de condicionamientos estructurales de la sociedad (Bourdieu, 1990). O bien, dicho de otra forma: en la construcción de formaciones discursivas que configuran el sentido de las prácticas sociales ubicadas en las fronteras de lo pensable, en donde los enunciados se escenifican en una serie de regulaciones para interpretar los hechos sociales (Foucault, 1968, 2004; Vasilachis de Gialdino, 2005).

De tal modo, reconstruir los nudos discursivos sobre la cuestión social que realiza un agente gubernamental revestido de legitimidad estatal, resulta pertinente para abordar el engranaje en las maneras en qué se problematizan los problemas sociales y se interviene sobre ellos en el marco del ejercicio del poder político. Es decir, recuperar esos fundamentos normativos que, si bien pueden no estar explicitados en la letra de la resolución, funcionan como orientadores que estructuran su formulación en el 
marco de una razonabilidad política. Por ello, se puede decir, que el discurso público gubernamental forma y conforma la constitución de un dispositivo estatal productor y reproductor del mundo, configurador y promotor de ciertas prácticas, el cual es ejercido mediante las legalidades asignadas dentro de una sociedad en el marco de la presentación del reconocimiento y/o prescripción de ciertos elementos formativos y reguladores, en este caso específico, de la intervención social del Estado.

En síntesis, se cree que la metodología empleada permitió indagar acerca de la formulación del programa de transferencia condicionada y de su proceso de implementación a partir de un conjunto de perspectivas y saberes articulados. En este sentido, resulta pertinente resaltar que el objetivo no fue analizar la circulación y el diálogo entre ellos. Más bien, se pretendió relevar desde distintas ópticas, puntos comunes a fines de establecer sugerencias para el desarrollo de acciones estatales futuras. Por ello, la propuesta se insertó en la producción de conocimiento, inmersa en la gestión dada la pertenencia a un espacio que había sido unidad ejecutora, y luego, con el giro en los programas de transferencia condicionada durante el gobierno de la alianza de Cambiemos, unidad capacitadora y de acompañamiento a titulares con referencia institucional y subjetiva a la institución.

En este marco, existen algunas premisas por donde osciló esta propuesta metodológica que se explicita en marcos y referencias teóricas que construyeron los prismas singulares por los cuáles analizar los datos construidos. Dicho marco, funcionó como un punto de partida, dado que la indagación iba haciéndole cuestionamientos y a nosotros preguntas. En cierta forma, se convirtieron en los anteojos que se usan para interpretar el PHF y, a medida que se avanza, ir incorporando otras lentes. Para ello, se propone en el siguiente apartado una reconstrucción bibliográfica del marco teórico general que se usa como punto de vista particular.

\section{La política pública como acción estatal}

La política pública puede ser definida como una respuesta estatal a un problema público; es decir, a una dimensión de lo público socialmente problematizada (Bonicatto, 2017). Esto significa, que es un problema de agenda pública que lo procesa un actor estatal. De no existir procesamiento por parte de un actor estatal no podría definirse como política pública sino como problema público. Por lo que siempre implica una intervención estatal, una acción que intenta resolver o modificar dicho problema social.

Las acciones estatales, en cuanto contenido, pueden ser entendidas como productos de una serie de operaciones que el Estado realiza sobre recursos e insumos de carácter público; productos que, entregados a la sociedad, producen resultados e impactos (Sotelo, 
2012). Los problemas públicos están relacionados entre sí, por lo que la política pública también puede representarse como un conjunto de acciones, vinculadas a esa red de problemas. En este sentido entendemos que la política pública integra un programa, es decir, se inscribe en una propuesta más amplia que orienta la acción. El programa de gobierno y la política pública tienen una dimensión normativa: son portadoras de valores, intereses y objetivos. Dicha dimensión normativa se expresa en el intercambio de problemas (Matus, 2007) que se producen en su desarrollo, en tanto su competencia rige y afecta a la totalidad de intereses, comportamientos de individuos, grupos y organizaciones del territorio sobre el que el Estado reclama y ejerce su soberanía. Dicha competencia se despliega gracias a un factor de coerción -la autoridad investida de poder público representada por el actor gubernamental-, por lo que el acto público tiene carácter de obligatorio y el Estado tiene los medios para garantizar su acatamiento de forma legítima. Por lo que siguiendo a Vilas también se podría definir a las políticas públicas como un "conjunto de acciones de gobierno ejecutadas para alcanzar los fines hacia los que se orienta el ejercicio del poder político" (Vilas, 2011, p. 38).

Dicho de otra manera, la política pública comprende, entre otras cuestiones, concepciones político ideológicas que se reflejan en la definición de cuáles son los problemas que atraviesan a la sociedad y que, por lo tanto, serán objeto y parte del diseño de ella. Esto implica que la definición de política pública es situacional y multiactoral; está indefectiblemente vinculada a un momento, lugar y actores con intereses determinados (Bonicatto, 2017). Es un proceso conflictivo en donde confluyen distintas formas de entender el mundo, de definir los problemas a abordar por la política y la forma de hacerlo, por lo que el resultado de dicho proceso es, volviendo a Matus (2007), un intercambio de problemas en donde cada decisión beneficia a unos y perjudica a otros. Por ello, se sostiene que no siempre, ni necesariamente la asistencia estatal mediante los mecanismos de seguridad y protección social mejoran las condiciones materiales de existencia (Danani, 2017), ni generan procesos sociales de ampliación de lo posible y horizontes de realización de los deseos e inquietudes de las personas.

Sin embargo, se podría partir de la base de que en toda intervención social del Estado subyace una idea de bienestar social, a partir de la cual se construyen una serie de dispositivos, que actúan y hacen actuar a las personas implicadas (Carballeda, 2002; Donzelot, 2007). Se delimitan las causas y se proyectan posibles consecuencias y resultados sobre eso que se interviene, donde se disponen y distribuyen un conjunto de recursos simbólicos y materiales para atender el problema social estatizado. De esta manera, se hace referencia a la transformación de una inquietud pública a sustancia estatal, la cuál es fragmentada, definida y abordada en un momento determinado de 
la historia y se relaciona con los ciclos políticos que conducen los estamentos institucionales, pero que a la vez se desarrolla en conjunto con la institucionalización de los conflictos sociales. En síntesis, por intervención social del Estado, se va a entender al proceso de institucionalización de las demandas y conflictos, donde se ponen a disposición un conjunto de recursos, saberes, normativas y leyes, las cuáles se materializan a partir del diseño de un grupo de dispositivos y mecanismos, desde donde regulan los problemas socialmente problematizados e incorporados en la agenda gubernamental.

Dicho con otras palabras, la intervención social del Estado se configura en la construcción de dispositivos y mandatos gubernamentales que se constituyen en las tramas de la asistencia a partir de la delimitación de un conjunto de problemas sociales reconocidos por los actores institucionales como críticos sobre los cuales se debe actuar, cuya finalidad puede ser la creación y el condicionamiento de prácticas y subjetivaciones, o bien, la transformación de ciertos elementos estructurales de la sociedad que hacen a la obturación del pleno acceso a la ciudadanía. Así dicho, se constituye como una acción estatal en tanto política pública que se transforma en un campo determinado y en el ejercicio de poder legalizado, donde se delimita y escribe una noción singular del bien común.

Por tanto, en ese circuito de normativas, decisiones, ideas-fuerza y dispositivos institucionales que despliega el Estado en un determinado ciclo político, se constituye en la "elección" de cuáles son los problemas a tratar y en la delimitación de sus nudos críticos. Así en el periodo 2015-2019, mientras Cambiemos gobernó Argentina, como se verá en los siguientes apartados, delimitó como locus de la cuestión social ${ }^{3}$ a la pobreza y a su nudo crítico a la capacitación, o mejor dicho, a la falta de ella.

\section{Perseguir la pobreza}

Luego de la dictadura cívico militar en Argentina, la pobreza se tornó el eje central de las intervenciones sociales del Estado, donde no solo se profundizaron los mecanismos de asistencia ya existentes, sino que también se consolidó como un elemento cuantificable (Grondona, 2014; Vommaro y Daniel, 2013). Puntualmente, el auge de este doble tratamiento se encuentra en la década de 1990 en donde se la definió como sinónimo de la cuestión social, atravesada por un conjunto de discursos de especialistas y recomendaciones de organismos

3. Para el sociólogo francés Robert Castel, la cuestión social puede ser definida como "una aporía fundamental en la cual una sociedad experimenta el enigma de su cohesión y trata de conjurar el riesgo de su fractura. Es un desafío que interroga, pone en cuestión la capacidad de una sociedad (lo que en términos políticos se denomina una nación) para existir como un conjunto vinculado por relaciones de interdependencia" (Castel, 2002, p. 20). 
internacionales (Cortés y Kessler, 2013; Grassi, 2003; Murillo, 2008; Lo Vuolo y Barbeito, 1999). En aquel periodo, toda la batería de programas sociales focalizados y el sistema de protección social se constituyeron como elementos compensadores y subsidiarios de las medidas económicas respecto a las privatizaciones masivas de las empresas estatales, las flexibilizaciones del mercado de trabajo, el aumento de la pauperización de los sectores populares y el desempleo masivo. Una mirada similar de la visión del PRO -partido que condujo la coalición gobernante a partir de 2015-, sobre el lugar que debe tener el Estado en la vida pública y las regulaciones que se deben llevar adelante.

Desde la perspectiva de la fuerza política liderada por Mauricio Macri, se cree que lo que debe dejarse sin regulación es el mercado, mientras el Estado debe intervenir socialmente y de forma activa para reducir el impacto de los desajustes del libre albedrío económico (Vommaro, Morresi y Bellotti, 2015), a la vez que tiene que preparar a los individuos para cuando llegue el progreso que generará procesos de mayores posibilidades de inclusión social mediante el empleo que generan los privados - lo que denominó Cambiemos como "lluvia de dólares" o "lluvia de inversiones" - y, en menor medida, los emprendedores. Esto significa, de cierta forma, que mientras ello se gesta, se produce y se consolida, se deben generar acciones compensatorias para sostener la reproducción de la vida cotidiana de los sujetos, mediante la satisfacción de las necesidades de existencia. A la par que ellos, los destinatarios de la asistencia, deben prepararse para que, llegado el caso, puedan acceder al mercado de empleo. Se define la contraprestación del programa en relación al mérito que se debe realizar.

Cambiamos de un período asistencialista a una propuesta de inclusión real y de mediano y largo plazo. Claramente hay un período de transición, hasta que logremos generar los empleos genuinos, formales, de calidad, que les permitan tener ingresos a las familias que hoy la están pasando mal. Mientras tanto, como Estado, garantizamos unos ingresos mínimos [...] Vamos en la senda correcta de la economía. Por la situación en que asumimos y en que estaba el país, lleva un tiempo y un proceso, pero se han tomado medidas que muestran un giro definitivo en el giro de la economía. Hay muchas medidas que tienen que ver con la confianza, la institucionalidad de un país que sale al mundo a pedir inversiones, a mostrarse como un país confiable y seguro para invertir, para generar empleo. (Liotti, 2016)

Cambiemos, conceptualizó el locus de la cuestión social como sinónimo de pobreza 4 . Pobreza Cero, no solo se forjó como una promesa de campaña expresada en su plataforma electoral y un núcleo narrativo, sino también en su sustancia estatal. El objetivo central

4. Esto marca un quiebre respecto al periodo kirchnerista donde el foco de las intervenciones sociales del Estado estuvo vinculado a la búsqueda de la reducción de la desigualdad. Para profundizar se puede ver Kessler (2010), Danani (2016), Uribe (2018) y Rozas (2018). 
de la asistencia del Estado, estuvo enmarcada en perseguirla como preocupación que ponía en riesgo la cohesión de la sociedad. En tanto interrogante, aparecía la tensión entre las medidas económicas y las intervenciones sociales.

\begin{abstract}
Más allá de lo que tuve que anunciar que es un aumento en lo que es pobreza, yo nunca abandoné, ni vamos a abandonar como gobierno el trabajo por reducir la pobreza [...] Argentina es un país que necesita trabajar mucho para ir en camino de reducción de la pobreza. Pobreza Cero es una meta de muy largo plazo que quiere decir que tenemos que trabajar primero todos juntos y coordinadamente porque son muchas cosas las que tienen que transformar en el mediano y largo plazo para poder pensar que las familias dejen definitivamente la pobreza. (La Nación, 2019)
\end{abstract}

Una vez definido el problema, se deben definir sus causas. Una economía política de la problematización pública procesada por el Estado. Dicho de otra forma, se define el nudo crítico, ese causante estructural que se busca regular y transformar, dado que en el subyacen también otras contingencias que producen nuevos malestares y complejidades. Así, Stanley puntualmente habla sobre las causas de la pobreza:

Si pensamos que el trabajo y la educación son los pilares básicos para salir de la pobreza, hicimos un giro en el programa y propusimos que terminen el secundario, con capacitación en oficios. Y está funcionando: padres que estudian y motivan a sus hijos a estudiar. (Spillman, 2018)

Entonces, por un lado, está el trabajo y, por el otro, la educación. El primero, como se vio, es responsabilidad de los privados, mientras que la capacitación es responsabilidad del Estado. Este último punto, se materializó en el PHF desde el cuál se propuso una visión reeditada de las intervenciones estatales en relación con la pobreza, en donde se buscó transformar y generar nuevas prácticas sociales. La Ministra de Desarrollo Social de la Nación, lo mencionaba en su cuenta de Twitter como una de las "grandes" transformaciones en materia social:

Transformamos los Programas Sociales y creamos Hacemos Futuro, un programa con foco en la persona. Todos los que son parte terminan sus estudios y se capacitan, porque la formación es una herramienta clave para la inserción en el mundo del trabajo. (@CaroStanley, 6 de diciembre de 2019)

El mismo, unificó a tres ya existentes: Ellas hacen, Argentina Trabaja y Desde el barrio. El acto institucional que da vigencia al nuevo programa se realiza el 8 de febrero de 2018 mediante la Resolución 96/2018. Sin embargo, su objetivo general lo encuentran en la Resolución 151/2018 de la Secretaría de la Economía Social del Ministerio de Desarrollo Social, donde se destaca: 
Empoderar a las personas o poblaciones en riesgo o situación de vulnerabilidad social, promoviendo su progresiva autonomía económica a través de la terminalidad educativa y cursos y prácticas de formación integral que potencien sus posibilidades de inserción laboral e integración social. (Resolución 151, 2018, p. 1)

Primera cuestión normativa a destacar de acuerdo al objetivo principal del programa de transferencia condicionada elaborado en 2018: la homologación entre formación educativa y autonomía económica. Resulta algo así, como un recurso subjetivado por diferentes miradas y sectores de la sociedad, desde donde pensar las intervenciones para reducir la pobreza, pensando específicamente en las acciones orientadas a los sujetos pobres. La propuesta pondera, entonces, que hacía mayores construcciones de capacidades cognitivas e instrumentales de las personas, más aumentan las oportunidades que se le abrirán en el mercado de trabajo porque mejoran las condiciones de empleabilidad. Es, se podría decir, la hipótesis que persigue esta política pública. Ella, se consolida como el fundamento simbólico que tiene la fuerza social devenida en gobierno. "La ayuda o el acompañamiento del Estado tiene que ser por un tiempo [...] lo acompañamos en tanto y en cuanto esa persona pueda hacer cosas donde se pueda ir superando" (La Nación, 2019).

La idea de temporalidad y superación individual, en este caso, creemos que puede resumir lo expuesto durante todo este apartado y podría exponerse de la siguiente manera. El Estado debe intervenir socialmente, mientras que el mercado, ya sin las cargas impositivas y con un país confiable -como dijeron los dirigentes de Cambiemos en más de una oportunidad-, invertirá para generar trabajo. Los sujetos, mientras tanto, se deben preparar y capacitar, superándose y generando las condiciones de empleabilidad. En este marco, se concentra una de las articulaciones entre el Estado y el mercado que propuso Cambiemos: mientras se espera que lleguen las inversiones, que se genere empleo de calidad que saquen a los individuos de la pobreza, ellos deben capacitarse; fortalecer el mérito, desde esa óptica, es una tarea estatal, para que estén preparados para cuando tengan la oportunidad.

\section{El individuo para "hacer futuro" 5}

Antes de iniciar con los objetivos y las intenciones de este apartado, a fines de clarificar, conviene resumir lo detallado anteriormente. Cambiemos definió dos cuestiones importantes en relación a las políticas públicas que hacen al área social del Estado. En

\footnotetext{
5. Este apartado recupera algunos elementos de un informe técnico realizado, en el marco de la Dirección de Gestión de Políticas Públicas de la Prosecretaría de Políticas Social de la Universidad Nacional de La Plata, de la cual somos parte. Para profundizar se puede ver Dirección de Gestión en Políticas Públicas (2019a; 2019b).
} 
primera instancia recortó el problema a tratar: la pobreza. Allí, se pusieron todos sus esfuerzos y se la catalogó como el locus de la cuestión social; es decir, como el eje estructurante socialmente problematizado e inquietud estatizada. También conceptualizó su nudo crítico: la falta de capacitación. Es decir, ese conjunto de prácticas, saberes y capacidades cognitivas e instrumentales que debían cambiarse para dar por terminado el problema. Promover la orientación educativa, mientras que el mercado hacía lo suyo y generaba trabajo de calidad. Las dos pinzas por donde salir de la encerrona.

En este sentido, como se menciona en la introducción, existe cierto consenso que en Argentina a partir de 2015 con el cambio de gobierno se produjo una transformación en el paradigma de la intervención social del Estado: se pasó del eje productivo al de la capacitación. Si bien, se podría decir que el trabajo y la educación figuran entrelazados en ambos periodos y de ninguna manera dejan de estar presentes en los fundamentos simbólicos de los programas, siempre hay uno que queda subsumido por el otro. En términos extremadamente generales y sin hacer juicios sobre si los objetivos perseguidos se lograron o no, se podría decir que el ciclo kirchnerista se caracterizó por promover y generar desde los programas sociales la creación de cooperativas y asociaciones de trabajadores excluidos del mercado formal de empleo a la vez que se ofrecían cursos para que realicen de acuerdo a las temas de interés, mientras que en el macrismo se buscó que los titulares se capaciten para mejorar las condiciones de empleabilidad y que se inserten al mercado laboral formal. En ambos casos, también en términos generales, se apoyan en sujetos diferentes: el primero suponía elementos relativos a la construcción de comunidad, de colectivos, a la vez que el segundo se hace eco de un sujeto más individualizado.

Ese giro, podría situarse en el intercambio de problemas que se habló anteriormente. O, mejor dicho, se estableció una nueva jerarquización normativa desde donde los agentes establecieron los nudos sustanciales por los cuáles atender el problema social estatizado. Para ser más precisos: ese cambio paradigmático en la definición de la contraprestación se constituye como una de las dimensiones que el ciclo político conceptualizó para la formulación de alternativas en relación a la pobreza, a fines de generar mejores condiciones de empleabilidad de los sujetos y se forjó como significante estatal en relación al mérito.

Al menos en el capitalismo, todas las sociedades bajo esta formación económica social definen sus concepciones en relación al mérito y, en el caso de los programas de transferencia condicionada, ello puede ser deducido por su contraprestación. Lo que cambia en el marco de los ciclos políticos, es el lugar que tiene y las prácticas sociales que lo constituyen.

Las alteraciones que subyacen en las concepciones del mérito para comprender las problemáticas sociales producto de la desigualdad persistente, se imprimen en dos 
primas: la igualdad de posiciones y la igualdad de oportunidades (Dubet, 2011). La adopción por una de ellas, cambia radicalmente el nudo explicativo y las acciones u omisiones que lleva adelante el Estado en las intervenciones sociales. En otras palabras, lo que se modifica es la concepción de justicia social que se adopta; las cuestiones vinculadas a las definiciones del bien común que realizan las fuerzas sociales en posiciones de gobierno. Cambiemos, respecto a ello, como explicaremos a continuación, adoptó una posición más afín a la igualdad de oportunidades, prisma que guarda estrecha relación con la meritocracia, en donde el PHF se constituyó como política de individuación.

\section{Fortalecer al individuo}

A partir de lo relatado por los titulares, investigadores y referentes de las organizaciones sociales, se va a argumentar que el PHF fue una política pública productora de individuación. Las diversas miradas se enredaron desde sus narrativas singulares para describir, caracterizar y conceptualizar esta política pública, respecto a las diferentes formas de vincularse con ella. Tanto desde los saberes técnicos, como de los cotidianos y las vivencias se escriben los diferentes aspectos, desde los relatos hasta los análisis, que permitieron indagar las cuestiones normativas, como sus materializaciones y producciones simbólicas. Se busca desde aquí, sintetizar ese puente que es el resultado del informe.

En términos generales, la unificación de los programas existentes y sintetizados en el PHF, tuvo en su primera instancia, algunos problemas relacionados a la escasa información que tenían los titulares. Sin demasiada difusión o eligiendo, quizás, los canales inadecuados para realizarla, se pudo relevar que hubo gran confusión sobre las características, los objetivos y requisitos que pretendía el Ministerio para otorgar y permanecer en el mismo. Por otra parte, también, hubo dificultades para acceder a los cursos, dado que no se encontraban "federalizados" y muchos "se concentraban en lugares" lejos de los espacios habitacionales de los titulares, lo que se interponía en muchas situaciones con tareas de la vida cotidiana y obturaba el cumplimiento de la contraprestación, lo que después resultó motivo de suspensiones.

Ello, en muchos casos, se forjaba como una barrera para el cumplimiento con la prestación del programa, dado que los titulares debían optar entre "ir a buscar a los chicos al colegio o cumplir con el programa”, pero sí no cumplían dejaban de recibir el dinero que en muchas situaciones les provocaba "angustia, desesperación e incertidumbre".

Otra de las cuestiones vinculadas al nuevo programa y al giro que constituyó el foco puesto en la capacitación, se encontró discusiones sobre la finalidad y el horizonte concreto que les modifique la vida cotidiana. Varios relatos resaltaban: 1) la dificultad de 
"entrar al aula" luego de "mucho tiempo"; b) la terminalidad educativa no les "garantiza" acceder al mercado de empleo; c) la "necesidad" de una dimensión que se enfoque en la promoción productiva para "reinsertarse" en el mundo formal del trabajo.

Ahora bien, se resume esta parte. La propuesta del gobierno de Cambiemos, se constituyó en un principio como un dispositivo de la incertidumbre, dado que se ponía en tensión con un horizonte posible para las personas. Es decir, que en la articulación entre el diseño del programa y las expectativas de los receptores no cabe una relación lineal. Más bien, se generó una zona gris generalizada donde la significación no encontraba imágenes concretas donde anclarse. Las voces narran un malestar, donde la proyección de lo concreto se desvanecía en la política pública que no fijaba un futuro situacional y posible. Recurriendo a la interpretación del gobierno sobre ello, los titulares debían esperar que "lleguen las inversiones", mientras debían mejorar sus condiciones de empleabilidad.

Volviendo ahora a las definiciones del programa, pero siguiendo por el mismo sendero analítico, es que resalta en sus definiciones ser un programa de carácter personal (Resolución 151, 2018, pp 1-2) a diferencia de las otras propuestas que unificó donde se incorporaban a las asociaciones familiares o comunitarias. Esta idea, llamó especialmente la atención de los saberes técnicos y especializados, para relacionarlo con la noción de individuación, definida en función del conjunto de los mecanismos y dispositivos que despliega el Estado, a partir de los cuales la integración social, en última instancia, se apoya en las voluntades individuales de los sujetos (Merklen, 2013). En este sentido, la individuación resultó como una racionalidad a fines de generar prácticas que devengan de la autonomía, la responsabilidad individual y la realización personal para estar preparados y enfrentar las reglas sociales de la sociedad.

Para esto, se constituye un andamiaje en la intervención social del Estado que persigue la hipótesis de que los problemas radican en las voluntades y actitudes, así como las acciones y racionalidades de los individuos. Allí, se pone al individuo como centro y pivot de la sociedad, ya que la creencia normativa que subyace es que la promoción y el desarrollo de las capacidades de cada persona es la que genera mayor bienestar social.

Pero aquí, se puede notar un doble juego interpretativo que se vinculó a los procesos normativos de Cambiemos: no solo se culpó a los sujetos, sino también al Estado por intervenir en los aspectos económicos y no dejar que el mercado se pueda desarrollar libremente y así generar empleos de calidad, ya sean con inversiones de empresas transnacionales o motivaciones emprendedoras ${ }^{6}$.

6. Este elemento fue, según Vommaro (2017), una de las motivaciones que motorizó a los Ceo's a "meterse en política" en la construcción de un pánico moral de una supuesta "chavización” de Argentina. 
La individuación de la política pública, también se constituyó como normativa para pensar la relación entre el Estado y los sujetos, a fines de fortalecer las intervenciones en la pobreza y promover nuevas prácticas sociales. Así, se condujo a un sistema centralizado en la Administración Nacional de la Seguridad Social (ANSES), apartando del lugar de entes ejecutores a las delegaciones municipales, las organizaciones de base territorial y otras instituciones civiles y estatales. De tal manera, se propone una relación individualizada de los titulares con el Estado, en tanto configuración normativa de la resolución de una propuesta anclada en la "persona".

Esto, según lo relevado, tuvo algunas consecuencias: 1) el "malestar" en los trabajadores de la burocracia del ANSES por "tiempos máximos" en la atención a los titulares; 2) desterritorialización y desintermediación de los programas; 3) desarticulación de las dependencias estatales y fragmentación de sus equipos técnicos; 4) "reforzamiento" de los imaginarios negativos y fracturación de las redes comunitarias vinculadas a los referentes barriales.

En este marco, otro de los elementos que nos interesa destacar en este trabajo, es que la información de cursos se centralizó en el portal "Formate en Red". Esta fue otra de las líneas que pueden ser evaluadas por dos motivos: las características de la población argentina, en donde la brecha digital atraviesa tanto por condición socioeconómica, como por localización geográfica; y porque la postulación de lo digital contiene una impronta de pretensión modernizadora propias de la gestión en el sector privado (Astarita, 2018). Sin embargo, utilizando datos del Instituto de Estadísticas y Censos (Indec) del país, se puede ver que el 19.7 \% de la población no cuenta con acceso a internet (Indec, 2018).

Este dato no es menor, si entendemos que como contraprestación para percibir el ingreso los titulares deben realizar cursos de capacitación que son difundidos por la web. Por ello, es que esta parte del diseño se constituyó como un limitante para el acceso a la información.

Parafraseando a los titulares, la experiencia virtual reside en el "mal" funcionamiento de la página. Siguiendo con la recuperación del relevamiento de las problemáticas que se suscitaron a partir del nuevo programa, se puede identificar: 1) escaso conocimiento de la población destinataria de la herramienta; 2) desactualización de los cursos que figuraban allí; 3) dificultad en el acceso. La propuesta modernizadora en el ámbito de la intervención social del Estado, terminó por resultar un limitante en tanto muchos de los titulares no contaban con acceso a las tecnologías, conocimiento o experiencia en su uso y tampoco hubo capacitaciones relativas a ello, a la vez que según los propios relatos de quienes sí tuvieron algún tipo de acercamiento, la plataforma no funcionaba de forma óptima. De la misma manera, esta promoción de los canales no presenciales, tanto en la limitación de los tiempos de atención de las agencias encargadas, como la información centralizada en internet retrató complejidades para sostener, en muchos casos, la titularidad del programa. 
En suma, si bien hubo ciertas estrategias realizadas por la Dirección de Seguimiento y Abordaje Territorial perteneciente al mencionado ministerio para convocar titulares del programa de forma particularizada, la promoción de la instancia virtual puede considerarse como un obturador para la permanencia del programa por parte de la población destinataria. Quizás, el problema no radicó tanto en su conformación sino en que no esté acompañado de otras instancias articuladas. Así, resultó "fundamental el acompañamiento" de instituciones que si bien, no tenían correspondencia formal con el programa, asesoraban y gestionaban los problemas que se le presentaban a las personas para el acceso y continuidad en él.

En síntesis, se entiende que en el programa operó simbólicamente una normativa individualizadora que configuró los aspectos materiales y simbólicos en la constitución de la intervención social del Estado durante el gobierno de Cambiemos. En esa enredadera se constituyeron los dispositivos, mecanismos y mandatos asistenciales desde los cuales se buscaron instituir la formación de capacidades cognitivas, morales e instrumentales para preparar a los sujetos, mientras llegaban las inversiones, o en su defecto, eran ellos quienes emprendían.

\section{Conclusiones}

Lo detallado durante todo el artículo, permite realizar algunas consideraciones finales. Reflexiones e interrogantes que quedaron resonando luego del análisis de los datos. En primer lugar, se cree que hubiese sido fundamental incorporar en la estrategia metodológica entrevistas en profundidad a trabajadores del Ministerio de Desarrollo Social de la Nación y a funcionarios políticos, para articular otras texturas interpretativas y argumentaciones sobre los objetivos del PHF, en el marco de la delimitación de los problemas que se buscaron resolver. Incluso, respecto a los segundos, habría aportado considerar más específicamente y con mayores niveles de profundidad y complejidad, las propias racionalidades y afectividades que devienen de las subjetivaciones políticas del proyecto gubernamental que persiguió Cambiemos en el área de la intervención social del Estado.

Por otra parte, se considera también, que las entrevistas utilizadas para reconstruir la perspectiva del agente institucional revestido de legitimidad legal para ejercer la función del cargo público como Ministra de Desarrollo Social, resultó una opción consistente de acuerdo al relevamiento que se propuso: comprender cuáles fueron las principales normativas dentro de esta gran área. Seguramente esa reconstrucción, no permitió rescatar las tensiones que tiene el complejo proceso de la política pública en clave multiactoral. 
En tercer término, la apuesta en grupos segmentados por saberes y vinculación al PHF, se cree que tuvo, en primer lugar, una fortaleza: todas las personas que se encontraron para debatir compartían visiones y recorridos similares, lo que permitió profundizar sobre las cuestiones más relevantes. También, hubiese sido pertinente, realizar grupos de discusión entre todos estos actores para abrir espacios a otros diálogos y debates posibilitados a partir de las diversas inscripciones en el mencionado programa.

Dicho todo ello, lo expuesto en estas páginas se sitúa de forma adecuada en el estado general del tema, dado que es un fenómeno de la historia reciente de Argentina. También, profundiza desde la reconstrucción de diversas posturas la intervención social del Estado durante el gobierno de Cambiemos.

En ese sentido, aporta algunos hallazgos. En primer lugar, la reconstrucción de la perspectiva del proyecto político personificado en Carolina Stanley desde donde se conceptualizó a la pobreza como núcleo estructural de la cuestión social y se llevaron adelante propuestas de asistencia vinculadas a la capacitación de las personas que se encuentran en esa situación. Lejos de una consideración sistémica y aclaraciones de acuerdo al sistema de acumulación, la novedad radicó en considerar como potencia inclusiva a la capacitación como vía para la construcción de herramientas morales, cognitivas e instrumentales. También allí, sobrevino una crítica y una adopción del lugar del Estado: promover la preparación de los sujetos, mientras que el mercado genera trabajo de calidad. Así también, se buscó la modernización de esta área centralizando la información en canales digitales, limitando las instancias presenciales de consulta y centralizando la atención en el ANSES.

Resumiendo, quedan latentes las críticas a este artículo para generar nuevos procesos de indagación, preguntas y problemas de investigación que se piensa, pueden aportar a conocer con mayor profundidad y complejidad la intervención social del Estado durante el gobierno de Cambiemos.

\section{Referencias}

[1] Ansaldi, W. (2017). Arregladitas como para ir de boda. Nuevo ropaje para las viejas derechas. Revista THEOMAI. Estudios críticos sobre Sociedad y Desarrollo, 35, 22-51. http:// revista-theomai.unq.edu.ar/NUMERO_35/2.\%20Ansaldi.pdf

[2] Arcidiácono, P. y Bermúdez, A. (2018). Del "Ellas Hacen" al "Hacemos futuro": descolectivización como impronta de los programas sociales. Revista de Políticas Sociales, 6(5), 65-72. http://www.rps.unm.edu.ar/ojs/index.php/rps/article/view/39 
[3] Astarita, M. (2018). Cambiemos y su modelo de Estado: Hacia un orden jerárquico y modernizador. En D. García Delgado, C. Ruiz del Ferrier y B. de Anchera (comp.), Elites y captura del Estado: control y regulación en el neoliberalismo tardío (pp. 61-89). FLACSO.

[4] Atria, J., Amenábar, J. Sánchez, J., Castillo, J. C. y Cociña, M. (2017). Investigando a la élite económica: lecciones y desafíos a partir del caso de Chile. Cultura-hombresociedad, 27(2), 5-36. http://dx.doi.org/10.7770/cuhso-v27n2-art1244

[5] Bonicatto, M. (2017). Gestión Estratégica Planificada (GEP). Un método para la géstión en organizaciones públicas. EDULP.

[6] Bonicatto, M. (2019). Doble Vía. Aportes de la planificación estratégica situacional a la extensión estatutaria de la UNLP. Arte Editorial Servicoop.

[7] Bourdieu, P. (1990). Sociología y cultura. Grijalbo.

[8] Bourdieu, P. (2011). Las estrategias de la reproducción social. Siglo Veintiuno.

[9] Canelo, P. (2019). ¿Cambiamos? La batalla cultural por el sentido común de los argentinos. Siglo Veintiuno.

[10] Canelo, P. y Castellani, A. (2017). Informe de Investigación $\mathrm{N}^{\circ} 1$. Perfil sociológico de los miembros del gabinete inicial del presidente Mauricio Macri. Observatorio de las Elites Argentinas; Universidad Nacional de San Martín.

[11] Carballeda, A. J. M. (2002). La intervención en lo social: exclusión e integración en los nuevos escenarios sociales. Paídos.

[12] Castel, R. (2002). La metamorfosis de la cuestión social: una crónica del salariado. Paidós.

[13] Castellani, A. (2018). Lobbies y puertas giratorias. Los riesgos de la captura de la decisión pública. Nueva Sociedad, 276, 48-61. https://nuso.org/articulo/lobbies-ypuertas-giratorias/

[14] Cortés, R. y Kessler, G. (2013). Políticas, ideas y expertos en la cuestión social de la Argentina democrática (1983-2012). Revista de Indias, 73(257), 239-264. http:// dx.doi.org/10.3989/revindias.2013.010

[15] Danani, C. (2016). Las políticas públicas del área de desarrollo social durante los gobiernos de Néstor Kirchner y Cristina Fernández. Análisis, 12, 1-25. https://library. fes.de/pdf-files/bueros/argentinien/12771.pdf

[16] Danani, C. (2017). La gestión de la política social: un intento para aportar a su problematización. En M. Chiara y M. M. Di Virgilio (orgs.), Gestión de la política social. conceptos y herramientas (pp. 25-52). Universidad Nacional General Sarmiento.

[17] De Imaz, J. L. (1964). Los que mandan. Eudeba.

[18] Dirección de Gestión de Políticas Públicas. (2019a). Análisis del Programa Hacemos Futuro. La experiencia de la ex unidad ejecutora de la Universidad Nacional de La Plata. https:// unlp.edu.ar/frontend/media/83/25183/b51184afdd7fe31786d1f8aa8c5a0647.pdf 
[19] Dirección de Gestión de Políticas Públicas. (2019b). Aportes de la universidad pública a los desafíos de los programas sociales en la actual coyuntura. https://unlp.edu.ar/frontend/ media/82/25182/4382dea18cfc46e1c0fa3240fb7e2acf.pdf

[20] Donzelot, J. (2007). La invención de lo social. Ensayo sobre la declinación de las pasiones políticas. Nueva Visión.

[21] Dubet, F. (2011). Repensar la justicia social. Contra el mito de la igualdad de oportunidades. Siglo Veintiuno.

[22] Ferrari-Mango, C. (2019). La transformación de la política social en contextos de reconstrucción neoliberal. Del Argentina Trabaja - Programa Ingreso Social con Trabajo al Hacemos Futuro [presentación de ponencia]. XIII Jornadas de Sociología, Buenos Aires, Argentina.

[23] Ferrari-Mango, C. y Campana, J. (2018). Del "Argentina Trabaja - Programa Ingreso Social con Trabajo" y el "Ellas Hacen" al "Hacemos Futuro". ¿Integralidad o desintegración de la función social del Estado? Observatorio sobre Políticas Públicas y Reforma Estructural; FLACSO.

[24] Foucault, M. (1968). Las palabras y las cosas. Una arqueología de las ciencias humanas. Siglo Veintiuno.

[25] Foucault, M. (2004). El orden del discurso. Fábula Tusquets.

[26] García Wolf, N. y Santiago, F. (2017). El control de las políticas públicas en la Ciudad Autónoma de Buenos Aires. Un repaso a veintiún años de su autonomía. En C. Ruiz del Ferrer (comp.), El control de las políticas públicas. La cuestión de la transparencia y la transparencia en cuestión (pp. 97-119). FLACSO.

[27] Gené, M. (2014). Sociología política de las elites. Apuntes sobre su abordaje a través de entrevistas. Revista Sociologia e Política, 22(52), 97-119. http://dx.doi. org/10.1590/1678-987314225207

[28] Gené, M. (2018). Politización y controversias: los CEOS en el gobierno de Cambiemos. Ensambles, 5(9), 41-62. http://www.revistaensambles.com.ar/ojs-2.4.1/ index.php/ensambles/article/view/143

[29] Gessaghi, V., Landau, M. y Luci, F. (2020). Clase alta, empresa y función pública en Argentina. Revista Mexicana de Sociología, 82(2), 403-428. http://mexicanadesociologia. unam.mx/index.php/v82n2/412-v82n2a6

[30] Grassi, E. (2003). Políticas y problemas sociales en la sociedad neoliberal. La otra década infame. Espacio.

[31] Grondona, A. (2014). Saber de la pobreza. Discursos expertos y subclases en la Argentina entre 1956 y 2006. CCC Centro Cultural de la Cooperación Floreal Gorini; CLACSO.

[32] Hopp, M. (2018). De la promoción del trabajo cooperativo al salario social complementario. Transformaciones en la transferencia de ingresos por trabajo 
en la Argentina. Ciudadanías. Revista de Políticas Sociales Urbanas, 2, 113-142. http:// ciudadanias.untref.edu.ar/pdf/n2_dossier_art5.pdf

[33] Hudson, J. P. (2018). Políticas públicas de promoción de la autogestión cooperativa de la Alianza Cambiemos. Perspectivas de Políticas Públicas, 8(15), 173-205. https://doi. org/10.18294/rppp.2018.2085

[34] Instituto Nacional de Estadística y Censos (Indec). (2018). Acceso y uso de tecnologías de la información y la comunicación. EPH. https://www.indec.gob.ar/uploads/informesdeprensa/ mautic_05_19CF6C49F37A.pdf

[35] Kessler, G. (2018). Exclusión social y desigualdad ¿nociones útiles para pensar la estructura social argentina? Lavboratorio. Revista de Estudios sobre Cambio Estructural y Desigualdad Social, 28, 4-18. https://publicaciones.sociales.uba.ar/index.php/ lavboratorio/article/view/105

[36] La Nación. (2019, mayo 16). Las 10 verdades de Carolina Stanley [Video]. Youtube. https://www.youtube.com/watch?v=wgqJZkYTtFs

[37] Landau, M. (2015). No sólo de globos vive el PRO: el macrismo en la larga tradición del gobierno de la Ciudad. Revista Ciencias Sociales, 87, 74-78.

[38] Liotti, J. (2016, 27 de noviembre). Stanley: "no se resuelve sólo con crecimiento la pobreza estructural". La Nación. https://www.lanacion.com.ar/politica/stanley-elcrecimiento-solo-no-resuelve-la-pobreza-estructural-nid1960001/

[39] Lo Vuolo, R. y Barbeito, A. (dirs.). (1999). La pobreza... de la política contra la pobreza. Miño y Dávila.

[40] Matus, C. (2007). Teoría del juego social. UNLa.

[41] Merklen, D. (2013). Las dinámicas contemporáneas de la individuación. En R. Castel, G. Kessler, D. Merklen y N. Murard (comp.), Individuación, Precariedad, Inseguridad: ¿desinstitucionalización del presente? (pp. 45-86). Paidós.

[42] Mills, C. W. (1957). La élite del poder. Fondo de Cultura Económica.

[43] Ministerio de Desarrollo Social de la Nación Argentina. (2018, 8 de febrero). Resolución 96/2018.

[44] Mosca, G. (1984). La clase política. Fondo de Cultura Económica.

[45] Murillo, S. (2008). Producción de pobreza y construcción de subjetividad. En A. Cimadamore y A. Cattani (coords.), Producción de pobreza y desigualdad en América Latina (pp. 41-77). CLACSO.

[46] Natanson, J. (2018). ¿Por qué? La rápida agonía de la Argentina kirchnerista y la brutal eficacia de una nueva derecha. Siglo Veintiuno.

[47] Nazareno, M. (2019). Neoliberalismo profundo. Apuntes sobre el "proyecto hegemónico” de la nueva derecha argentina. En M. Nazareno, M. S. Segura y 
G. Vázquez (eds.), Pasaron cosas: política y políticas públicas en el gobierno de Cambiemos (pp. 23-44). Brujas; Universidad Nacional de Córdoba.

[48] Pareto, V. (1968). The Rise and Fall of Elites. An Application of Theoretical Sociology. Bedminster Press.

[49] Rozas Pagaza, M. (2018). La Cuestión Social: su complejidad y dimensiones. Concienciasocial. Revista Digital de Trabajo Social, 2(3), 45-56. https://revistas.unc.edu. ar/index.php/ConCienciaSocial/article/view/21587

[50] Salerno, A. (2017). Evitismo liberal: voluntarias y peronistas en el Ministerio de Desarrollo Social de CABA durante la gestión PRO (2007-2015). Papeles de Trabajo, 11(20), 167-188. https://dialnet.unirioja.es/servlet/articulo?codigo=6232702

[51] Salerno, A. (2019). Una nueva derecha embarrada: perfiles de gestión PRO en torno a la cuestión social de la ciudad de Buenos Aires (2007-2015). Sociohistórica, 44, 1-19. http://sedici.unlp.edu.ar/handle/10915/88137

[52] Secretaría de Economía Social, Ministerio de Desarrollo Social de la Nación Argentina. (2018, 15 de febrero). Resolución 151/2018.

[53] Soler, L., Giordano, V. y Saferstein, E. (2018). Las derechas y sus raros peinados nuevos. Apuntes de Investigación del CECYP, 30, 171-191. http://apuntescecyp.com.ar/ index.php/apuntes/article/view/689

[54] Sotelo, J. (2012). Cadena de valor público y planteamiento estratégico, limitaciones y virtudes del modelo [presentación de ponencia]. XVII Congreso Internacional del CLAD sobre la Reforma del Estado y de la Administración Pública, Cartagena, Colombia.

[55] Spillman, E. (2018, 29 de julio). Carolina Stanley: "tenemos que trabajar para desterrar la extorsión en la calle, que son los piquetes". Perfil. https://www.perfil.com/noticias/politica/ tenemos-que-trabajar-para-desterrar-la-extorsion-en-la-calle-que-son-los-piquetes.phtml

[56] Stanley, C. [@CaroStanley] (2019, 6 de diciembre). Transformamos los Programas Sociales y creamos Hacemos Futuro, un programa con foco en la persona. Todos los que son parte terminan sus estudios y se capacitan, porque la formación es una herramienta clave para la inserción en el mundo del trabajo. Twitter. https://twitter.com/carostanley/ status/1203012149402120192?lang=ar

[57] Uribe Gómez, M. (2018). Ciclos políticos y política social en América Latina en el siglo XXI. Forum, 13, 101-118. https://doi.org/10.15446/frdcp.n13.70491

[58] Vasilachis de Gialdino, I. (1997). La construcción de representaciones sociales: el discurso político y la prensa escrita. Gedisa.

[59] Vasilachis de Gialdino, I. (2005). La representación discursiva de los conflictos sociales en la prensa escrita. Estudios Sociológicos, 23(67), 95-137. https://www. redalyc.org/pdf/598/59811838004.pdf 
[60] Vilas, C. (2011). Política y políticas públicas en América Latina. En C. Fioramonti y P. Amaya (comp.), El Estado y las políticas públicas en América Latina (pp. 37-74). AECID; COPPPAL; Honorable Cámara de Senadores de la Provincia de Buenos Aires.

[61] Vommaro, G. (2017). La larga marcha de Cambiemos: la construcción silenciosa de un proyecto de poder. Siglo veintiuno.

[62] Vommaro, G. y Daniel, C. (2013). ¿Cuántos son los pobres? Contribuciones a la historia de su definición estadística en la Argentina de los años ochenta. Voces del fénix, 23(4), 24-31.

[63] Vommaro, G., Morresi, S. y Bellotti, A. (2015). Mundo PRO. Anatomía de un partido fabricado para ganar. Planeta.

[64] Voria, M. A. y Míguez, M. E. (2019). Controversias en torno a la terminalidad educativa y la empleabilidad de mujeres destinatarias de programas sociales: virajes de gestión en torno al programa Hacemos Futuro. STUDIA POLITICA, 47, 129-167. https://doi.org/10.22529/sp.2019.47.06

[65] Zícari, J. (2016). Las coaliciones neoliberales en la Argentina: los casos de la Alianza y Cambiemos. Realidad Económica, 307, 6-36. http://www.iade.org.ar/system/files/ articulos/2_zicari.pdf 